\title{
SALUD
}

\section{Comportamientos de riesgo para transmisión de VIH en mujeres indígenas del Paraguay. Año 2016}

\author{
Analía Ortiz¹, Gloria Aguilar1,2, Gladys Estigarribia', Anibal \\ Kawabata², Sergio Muñoz ${ }^{3}$, Adele Schwart Benzaken, \\ Angélica Miranda Espínoza ${ }^{5}$
}

\section{Resumen}

Introducción: La Organización Mundial de la Salud (OMS), estima 10,6 millones de casos nuevos de sífilis por año, siendo una causa importante de preocupación para la salud pública, debido a las consecuencias que esto implica para la salud sexual y reproductiva de las mujeres. En el Paraguay la prevalencia de sífilis en puérperas fue del $4 \%$ en el año 2012. La epidemia mundial de VIH tiene un impacto desproporcionado en los grupos marginados de personas, las minorías raciales y étnicas, incluidos los pueblos indígenas. Las desigualdades socioeconómicas que enfrentan los pueblos indígenas incluyen: la pobreza, el uso indebido de sustancias, la falta de vivienda y el acceso desigual a la atención de salud conllevan un mayor riesgo de infección por VIH A pesar de la evidencia de una mayor vulnerabilidad al VIH entre las mujeres indígenas, pocas estrategias de prevención son sensibles al género, y aún menos se han centrado en las mujeres indígenas. En este contexto, se realiza esta investigación para analizar determinar los comportamientos de riesgo que predisponen a la trasmisión de sífilis en mujeres indígenas en el Paraguay.

Objetivo: Determinar los comportamientos de riesgo para transmisión de sífilis en mujeres indígenas de 5 familias lingüísticas del Paraguay en el 2016.

Material y Métodos: Se realizó un estudio diseño observacional, descriptivo de corte transversal con componente analítico, y muestreo probabilístico estratificado multietapico sobre la prevalencia de sífilis y factores de riesgo asociado mujeres indígenas de 5 familias lingüísticas.

Fueron incluidas mujeres pertenecientes a la población indígena de las 5 familias lingüísticas, de 15 y más años de edad que hayan accedido a participar del estudio firmando un consentimiento informadoSe realizó el test rápido de sífilis como tamizaje inicial a todas las personas que ingresaron al estudio, se

\footnotetext{
1. Universidad Nacional de Caaguazú, Paraguay.

2. Programa Nacional de Lucha contra en Sida, Paraguay.

3. Universidad de la Frontera-Facultad de Medicina, Temuco, Chile.

4. Fundación Alfredo da Matta, Brasil.

5. Universidad Federal do Espíritu Santo, Vitoria, Brasil.

E-mail: Analia.ortizrolon@gmail.com

DOI: $10.26885 /$ rcei.foro.2017.41
} 
utilizó prueba rápida (treponémica) y en el caso de resultado positivo se realizó la toma de muestra por punción venosa y realización del VDRL cuantitativo. Fueron respetados los principios de confidencialidad, igualdad y justicia. El protocolo de investigación fue aprobado por el comité de ética institucional del Instituto de Medicina Tropical.

Los datos obtenidos por las encuestas y los resultados serológicos fueron analizados mediantes el software STATA $14.0^{\circ}$. Los datos se expresan en medidas de tendencia central, dispersión y proporciones, para la comparación de los grupos se consideró una $p<0,005$.

Resultados: Se incluyeron al estudio 1872 mujeres indígenas en edad fértil pertenecientes a 5 familias lingüísticas, la prevalencia global hallada es de 6,68\%, correspondiendo a un $7,5 \%(77)$ a la familia guaraní $(p=0.05)$, la prevalencia de sífilis en el grupo etario de 30 a 39 años fue de 7,42\% (32), y el grupo etario de 30 a 39 años \%(32), no existen diferencias estadísticamente significativa entre los distintos grupos etarios, de acuerdo a la región la prevalencia en la región oriental fue de $7,25 \%(76)(p=0,055)$, respecto a las familias, en los guaraníes el $7,5 \%(77)(p=0,008)$. En cuanto a la prevalencia de acuerdo a la edad de inicio de relaciones sexuales, el 7,35\% (48) fue menor o igual a 14 años ( $p=0,336)$, el número de parejas 2 y más parejas registro $11,60 \%(21)(p=0,005)$ y el grupo de sexo a cambio de dinero 9,23\%(18) ( $p=0,004)$.

Conclusión: Se encontró una asociación estadísticamente significativa entre la prevalencia de infección y la región, el número de parejas sexuales y sexo a cambio de dinero. Las intervenciones para reducir los comportamientos sexuales asociados con un mayor riesgo de Infecciones de transmisión sexual en mujeres indígenas deben llevarse a cabo junto con los programas destinados a abordar el sexo transaccional, enmarcados en el respeto a la interculturalidad y la cosmovisión indígena.

Palabras clave: factores de riesgo, sífilis, vih, sida, población indígena.

\section{REFERENCIAS}

Ministerio de Salud Pública y Bienestar Social. Programa Nacional de Control de $\mathrm{VIH} /$ sida/ITS. (2016). Informe de la situación Epidemiológica de VIH, sida y sifilis, Paraguay 2016. Asunción: MSPyBS.

Sameer, B. (2017). Factores de riesgo en Canadá para enfermedades de transmisión sexual y sus variaciones/Risk Factors For Sexually Transmitted Diseases in Canada and Provincial Variations. Medicina Social, 11(2), 58-65.

World Health Organization. (2012). Strategies and laboratory methods for strengthening surveillance of sexually transmitted infection 2012. Switzerland: WHO. 